\title{
Streamlining the dental care during COVID-19 pandemic: updated clinical recommendations and infection control management framework
}

\section{Luciano José PEREIRA ${ }^{(a)}$ \\ Ramiro Mendonça MURATA ${ }^{(b)}$ Vanessa PARDI(b)}

Flávio Freitas MATTOS(c)

\footnotetext{
(a) Universidade Federal de Lavras - UFLA, Department of Health Sciences, Lavras, MG, Brazil.

(b) East Carolina University - ECU, School of Dental Medicine, Department of Foundational Sciences, Greenville, SC, United States of America.

(c) Universidade Federal de Minas Gerais - UFMG, Dental School, Department of Social and Preventive Dentistry, Belo Horizonte, MG, Brazil.
}

Declaration of Interests: The authors certify that they have no commercial or associative interest that represents a conflict of interest in connection with the manuscript.

\section{Corresponding Author:}

Luciano José Pereira

E-mail: lucianopereiraufla@gmail.com

hitps://doi.org/10.1590/1807-3107bor-2021.vol35.0046

Submitted: November 12, 2020

Accepted for publication: January 4, 2021

Last revision: February 8, 2021

\begin{abstract}
Several guidelines for dental practice have been published by dental associations and regulatory boards since the beginning of the pandemic. Initially, all non-emergency dental treatment were suspended. Healthcare personnel around the world are gradually expanding back to elective procedures. International updated recommendations alert that professionals must maintain regular observation of local health department reports, ensure personal protective equipment, and screen all patients for COVID-19 signs and symptoms. Telehealth strategies, patient screening, rescheduling when presenting COVID-19 symptoms and/or history of contact with infected people and hand/environment hygiene practices are reinforced. Appropriate cleaning and surface disinfection are mandatory. The dental staff must be trained to use appropriate Personal Protective Equipment (PPE), following a risk assessment and standard precautions: gloves; fluid resistant disposable gown, eye protection (face shield or goggles) and a medical mask. A fit tested N95 or KN95 respirator (or higher) is recommended when aerosol generating procedures are performed. Only essential accompanying persons should attend to dental appointment with the patient and must wear a cloth face covering or facemask. Social distancing and mask wearing by all staff are necessary in all areas of the office. Dental health care providers should keep up to date to the current guidance of clinical practice during the pandemic.
\end{abstract}

Keywords: Oral Health; Coronavirus; Health Policy; COVID-19.

\section{Introduction}

Since December 2019, dentistry is facing a new turning point. Dental schools, regulatory boards, scientific associations, government authorities, and public and private health care services joined efforts to design enduring answers for increasingly severe and long-standing viral challenges. ${ }^{1,2}$ Dentists took part as first line professionals since they work in close contact with patients and are exposed to the risk of being affected by COVID-19 and all respiratory infections. Due to the nature of dental procedures and the large number of droplets and aerosols which could be generated, standard protective measures in 
daily dental clinical work are not effective enough to prevent the spread of COVID-19.3

Several guidelines for dental practice environments have been published initially recommending the suspension of non-emergency dental treatment while providing emergency dental services only, ${ }^{4,5}$ Recent interim guidances to advise dental professionals who are returning to work after practice restrictions brought by COVID-19 have been published by the Advisory Task Force on Dental Practice Recovery of the American Dental Association (ADA) and Centers for Disease Control and Prevention (CDC) ${ }^{6,7}$ Dentists are returning to their practice routines at different times, depending on several conditions. Each team/institution will need to make decisions considering their knowledge of the local prevalence of COVID-19 cases, patients' needs, and the availability of supplies, before re-engaging in the provision of routine oral health care.

International experiences in dealing with communicable diseases are useful references for governments, professional associations, and policy makers. Public health actions must be supported by social media both in preparedness and controlling the disease. They can team up with government to provide consistent, simple, and clear messages. Quick information, either negative or positive, influence the public. Correct, short, and motivational community messages are fundamental in achieving public and professional acceptance and support of COVID-19specific control measures. ${ }^{8}$

In 2019, the World Health Organization (WHO) published the policy paper Building Bridges in Preparedness. ${ }^{9}$ It reported results of a 2017 meeting in which stakeholders proposed guiding principles to strengthen countries preparedness for pandemic influenza and health security. It was recommended that individual countries should develop national strategic multisectoral collaboration, take a holistic approach in developing national health emergency preparedness, include all relevant strategic and operational partners in key activities, take immediate action to ensure up-to-date plans, and develop a long-term strategy to test, review and periodically update preparedness plans based on the latest experience and expertise. Countries should also enable optimal collaboration before, during, and after public health emergencies, including harmonizing all plans, procedures, and tools. Global coordination and monitoring mechanisms should boost the availability of vaccines. WHO should support countries to construct multisectoral collaboration and partnership aiming at pandemic preparedness, develop strategies for helping countries to transform plans into actual capacities and actions, and support countries to implement, test, and update their preparedness plans. ${ }^{9}$

Different countries and even regions inside large countries are currently at diverse stages of the pandemic. Local health systems are the most important evaluation parameters in order to establish better working approaches to each place ${ }^{10}$. Although extremely efficient, social isolation is unsustainable in the long run and the identification of asymptomatic and presymptomatic carriers of SARS$\mathrm{CoV}-2$ is the strategy that allows progressive return to daily activities. Until a vaccine is available, SARSCoV-2 may continue to cause outbreaks requiring new rounds of social closure and protection to susceptible groups. ${ }^{10,11}$

\section{Update on centers for disease control and prevention (CDC) recommendations}

Previously, CDC have provided recommendations for dentists to prioritize urgent visits and delay elective care during the pandemic. ${ }^{4}$ However, on June $30^{\text {th }}, 2020$, a new Framework for Healthcare Systems Providing Non-COVID was published. ${ }^{12}$ This document reported the need of health professionals to get back to necessary services, but still respecting local level of COVID-19 transmission and considering changes over time. In summary, this CDC new statement indicates that healthcare personnel may gradually expand to elective services but must maintain regular observation of local health department reports, ensure personal protective equipment, and screen all patients for COVID-19 signs and symptoms. In addition, professionals should prioritize patients with unavoidable needs (services that, if postponed, may 
cause patient harm), at-risk populations and people without access to telehealth. ${ }^{12}$

The Interim Infection Prevention and Control Recommendations for Healthcare Personnel During the Coronavirus Disease 2019 (COVID-19) Pandemic was also updated on July $15^{\text {th }}, 2020$. Recommendations were divided into: a) Infection prevention and control (IPC) practices for routine healthcare delivery during the pandemic; and b) Recommended IPC practices when caring for a patient with suspected or confirmed SARS-CoV-2 infection. ${ }^{13}$ The authors mentioned that changes were not substantial, except for the added language informing that protective eyewear with gaps between glasses and the face likely do not protect eyes from all splashes and sprays. ${ }^{13}$ In the first section, telehealth strategies, patient screening, rescheduling when presenting COVID-19 symptoms, hand hygiene practices and cloth face covering before starting treatment are reinforced. Healthcare professionals should also use facemasks and face shields in order to avoid exposure to splashes and sprays and face touching. Eye protection and an N95 or equivalent or higher-level respirator are indicated during aerosol generating procedures. Once more, CDC recommends that professionals should consider the potential for patient harm if care is deferred about providing elective procedures. On the other hand, when caring for a patient with suspected or confirmed SARS-CoV-2 infection, these must be referred to health units exclusively dedicated to this end, and professionals must communicate with public health authorities. Appropriate cleaning and surface disinfection are also mandatory associated to N95 or equivalent or higher-level respirator, gown, gloves, and eye protection. Cloth face coverings are not considered Personal Protective Equipment (PPE) and should not be worn for the care of patients. ${ }^{13}$

Lastly, on August $28^{\text {th }}$ the CDC updated the Guidance for Dental Settings (Interim Infection Prevention and Control Guidance for Dental Settings During the Coronavirus Disease 2019 (COVID-19) Pandemic). ${ }^{7}$ This document reported specific infection control considerations, prioritizing the most critical dental services to minimize harm to patients and potential exposure toSARS-CoV-2 infection, reinforced the need to stay home if sick (to both patients and dental staff) and procedures to be adopted during contact with symptomatic patients.

In general, when in contact with patients (including those not suspected of SARS-CoV-2 infection), all staff must wear eyes, nose, and mouth protection. N95 respirator or a respirator that offers an equivalent or higher level of protection is mandatory for aerosol generating procedures. Indeed, CDC recommends that: dental elective procedures should be postponed (unless in unavoidable situations), prioritize treatment of patients at most risk if care is delayed, and the use of teledentistry screening. Only essential accompanying persons should attend to dental appointment with the patient and both must wear a cloth face covering or facemask until the beginning of treatment. Patients with suspect COVID-19 should wait until the end of isolation (virus incubation period). Patients with symptoms of COVID-19 should not receive non-emergency dental care. $^{7}$

Body temperature from all patients (and accompanying person) should be screened (fever if $\geq 37.8^{\circ} \mathrm{C}$ ) before entering the dental office. Besides, they should be asked about self-quarantine due to exposure to someone with COVID-19. Dental staff must provide visual alerts considering respiratory and cough hygiene measures, besides to alcoholbased hand rub (ABHR) with at least $60 \%$ alcohol, tissues, and no-touch receptacles for discarding. All unnecessary objects in the waiting room should be removed (e.g. toys, magazines) ${ }^{7}$

Staff members should frequently monitor themselves for COVID-19. If one of them presents fever or other signs and symptoms, he or she must return home, and seek medical evaluation. Occasional testing of asymptomatic patients and members of the dental team might be useful considering availability and possibility of negative results during virus incubation period. When conducting aerosol generating procedures, four-handed dentistry is preferable, along with preprocedural mouth rinses (e.g. hydrogen peroxide or povidone-iodine), high suction, and ruber dam to reduce droplet spatter and aerosols. ${ }^{7}$ Environmental cleaning and disinfection within patients should be carried out constantly. ${ }^{14}$ Emergency dental care for COVID-19 
patients or suspect should follow CDC's Interim Infection Prevention and Control Recommendations for Healthcare Personnel during the Coronavirus Disease 2019 (COVID-19) Pandemic described above. ${ }^{13}$ Aerosol generating procedures should ideally take place in an airborne infection isolation room. All staff members must wear a N95 or equivalent or higher-level respirator, goggles or a face shield, gloves, and a gown. ${ }^{7}$

\section{Update on the American Dental Association (ADA) recommendations}

The American Dental Association (ADA) has developed an interim guidance toolkit ${ }^{6}$ for dental health care providers (DHCP) for delivery of elective dental care during COVID-19 pandemic. The document was focused on the short-term management of dental practice during the pandemic as offices return to providing routine oral health care. The ADA considerations for the provision of non-emergency oral health services in the context of COVID-19 will be described below.

Screening of patients before their appointments by either video conference, virtual/remote technology or telephone is recommended ${ }^{6}$. The aim is to ensure that patients have no symptoms suggestive of COVID-19 infection or previous risk exposure [e.g.: fever or feverish recently (14-21 days); shortness of breath; cough; flu-like symptoms; loss of taste or smell; contact with confirmed COVID-19 positive patients; age over 60; systemic disease (diabetes, heart disease, lung disease, kidney disease or autoimmune disorders); traveled in the past 14 days. It is important to note that not all people infected with SARS-CoV-2 exhibit symptoms, and cases without symptoms can transmit to others. The staff performing virtual triage should inform that all who enter the office will be asked to wear masks except when undergoing treatment and remind patients to limit extra companions on their trip to your office to only essential people in order to reduce the number of people in the reception area.

Dental office must provide a hand sanitation station upon entry into facility. Inform that all patients/ guardians should wear masks. ${ }^{6}$ The waiting area must have alcohol-based hand sanitizer (greater than $60 \%$ ethanol or $70 \%$ isopropanol alcohol), tissues and chairs placed 6 feet (1.82 meters) apart when is possible. Reading material, remote controls, toys and other communal objects must be removed or cleaned regularly.

DHCP performing triage on site should maintain physical distancing, wear medical mask, check patient's temperature (touchless forehead scanner), complete patient screening form, and confirm no symptoms of COVID-19 or risk exposure. ${ }^{6}$ DHCP must provide materials to clean pens, clipboard, counter, phone, keyboards, light switches, surfaces, and anything else high touch. Post-op instructions should include a reminder to report any signs or symptoms of COVID-19 within next 2 days.

Dentists and staff should limit paperwork in the operatory room as much as possible. Limit access to the patient only, when possible. ${ }^{6}$ Supply a mask to anyone who accompanies the patient and keep staff level in operatory to the minimum required. Review overall health history and confirm that the screening questions were asked during the check-in procedure, and review if necessary. Decide on treatment using clinical judgment and known facts, combining patient health/risk factors/geographic incidence of COVID-19; procedural requirements/ clinical risks (production of aerosol, inducement of patient cough during procedure, ability to employ use of rubber dam); availability of PPE with relation to risk. Use professional judgment to employ the lowest aerosol-generating armamentarium when delivering any type of restorative or hygiene care.

For disinfection, clean the operatory while wearing gloves, a mask, and face shield or goggles. Dispose of surface barriers after each patient and use products that meet criteria for use against SARS-CoV-2 and are appropriate for the surface, following manufacturer's instructions. Until further evidence demonstrates a clear answer, the ADA did not make a recommendation on waiting for a specific time period to begin cleaning the operatory room.

The DHCP must be trained to use appropriate PPE, following a risk assessment and standard precautions: gloves; fluid resistant disposable gown, 
eye protection (face shield that covers the front and sides of the face or goggles) and a medical mask. ${ }^{6}$ A fit tested N95 or KN95 respirator (or higher) is recommended when aerosol generating procedures are performed. Social distancing and mask wearing by all office staff are necessary in all areas of the office, to the extent possible.

Front desk staff can wear masks and goggles, or face shields, or offices can install a clear barrier. Consider individual phone headsets for each front desk staffer to reduce virus spread through the phone hand piece. Private staff areas must be modified or adjusted to maintain social distancing of 6 feet between employees, where possible. Consider installing transparent shields or other physical barriers where possible to separate employees where social distancing is not an option. If seating cannot accommodate proper spacing, strive to manage scheduling to reduce the number of people in space at one time. Consider either installing no-touch activation faucets, providing individual bottled water, or encouraging staff to bring their own water to minimize touching of water faucets. Set a schedule for sanitizing high-touch surfaces in these areas as is also being done throughout facility. When possible designate separate bathrooms for use by staff and patients, making sure high touch areas (faucets, toilet handles, soap and towel dispensers, doorknobs, light switches) are cleaned often. Encourage staff to wipe down the bathrooms after each use.

Strict attention should be given to staff hand hygiene. Instruct staff to clean hands thoroughly: upon entry into the workplace; before and after any contact with patients; after contact with contaminated surfaces or equipment; upon entry to and exit from common private staff areas such as employee lounges, locker rooms, or lunch rooms.

Masks should be worn by staff at all times when in the office except when impractical. If available, gowns should be considered. Change gown if it becomes soiled. Disposable gowns should be discarded after use. Cloth gowns should be laundered after each use. If scrubs are to be worn, change between street clothes and scrubs upon entry and exit, or do the same with other office garb. Provide laundry facilities in the office. Contracting with a laundry service is another option.

Pregnant staff members should seek and follow medical guidance from their physician regarding work. Information on COVID-19 in pregnancy is very limited; offices may want to consider limiting exposure of pregnant staff to patients, especially during higher risk procedures (e.g., aerosol-generating procedures) if feasible, based on staffing availability.

Consider implementing a daily health screening check point and log for all employees entering the workplace. Ask all persons reporting to work the following questions, remembering to respect their confidentiality:

Do you have any of the following?

a. fever or feeling feverish;

b. shortness of breath (not severe); and

c. cough.

Persons who are well but who have a sick family member at home with COVID-19 should notify their supervisor. If an employee is confirmed to have COVID-19, the employer should inform fellow employees of their possible exposure to COVID-19 in the workplace but maintain confidentiality.

Recently ADA published a longitudinal study to estimate the prevalence of COVID-19 among US dentists. ${ }^{15}$ The web-based survey was designed to track infection control practices and infection rates within US dental offices. The study shown, as of June 2020, an estimated $0.9 \%$ (95\% CI) of US dentists have or have had COVID-19. Furthermore, $99.7 \%$ of dental offices adopted enhanced infection control protocols. These rates support that the use of $\mathrm{CDC}^{13}$ and $\mathrm{ADA}^{16}$ guidance on infection control procedures in dental offices will contribute to the reduced risk of SARS-CoV-2 infection during oral health care. Even though these are very compelling data this investigation has some limitations. The response rate was $40.1 \%$ and there is potential risk of recall bias among the participants. The web-based survey sample potentially have selection bias, leading to an underestimation of prevalence because dentists who have died or been hospitalized with COVID-19 did not or less likely participate on the survey. In addition, access to COVID-19 test were inadequate throughout 
United States and dentists might have undiagnosed disease. Despite these limitations, the web-based survey provides important evidence that incidence of COVID-19 among dentists is low and indicates that the current infection control recommendations may be sufficient to prevent SARS-CoV-2 infection in dental office.

\section{ADA recommendation if DHCP has a confirmed case of SARS-COV-2}

If a member of the staff has a confirmed diagnosis of COVID-19, he/she should follow all medical recommendations, including quarantine for 14 days to monitor symptoms. In addition, this person should seek medical treatment immediately if symptoms worsen and try to determine who may have had contact with him/her when still in the dental office. The office staff must be notified of the diagnosis and answer the following questions: When were they last in contact with the individual diagnosed with COVID-19? Get as detailed an answer as possible.

Some questions to ask include:

a. what was the date of the last contact?

b. how long was the contact?

c. what was the approximate distance of the contact?

d. are they experiencing symptoms? It's a good idea to instruct them to notify the other members immediately if they experience any change in symptoms.

The dentist or other member should conduct a risk assessment for any healthcare provider who was exposed to the individual with a confirmed case of COVID-19 so they can take steps, such as quarantining, seeking testing, and implementing any appropriate work restrictions. The unaffected staff may seek testing and keep the rest of the staff informed regarding the date tested, when they received results and what those results were, the progression of symptoms, any hospitalization or improvement. ${ }^{17}$

It is important to contact all patients who may have had contact with the COVID-19 positive individual to determine whether they are symptomatic. Those should be recommended self-quarantine for 14 days and notify the dental office if symptoms develop. ${ }^{17}$

All the recommendations of the CDC and local public health authority for additional steps might be followed..$^{13}$ See the CDC's Interim Infection Prevention and Control Recommendations for Patients with Suspected or Confirmed Coronavirus Disease 2019 (COVID-19) in Healthcare Settings for guidance on infection control in a healthcare setting.

\section{Update on the Brazilian Ministry of Health recommendations}

Brazilian Ministry of Health (MS) and the Brazilian Health Regulatory Agency- ANVISA (Agência Nacional de Vigilância Sanitária) have published technical notes on the provision of oral health care. ${ }^{18,19}$ They reassure that dental materials and procedures with aerosol production can spread the new coronavirus (SARS-CoV-2). These represent a risk not only to the dental team, as they can also trigger contamination without direct contact with infected Covid-19 people due to fluids in the air that contaminate the environment and surfaces. Risks are related to inadequate decontamination or sterilisation of environments and instruments. Training of DHCP on both subjects is essential for oral health care provision in an orderly and safe manner to patients and professionals.

For primary and specialised oral health care in the national health system, it is recommended to suspend elective dental care and to maintain emergency dental care. Whenever possible, prior remote contact is recommended to identify emergency dental cases. DHCP must ensure that users are neither left unattended nor suffer risks of SARS-CoV-2 contamination, from reception and clinical screening to symptom assessment, case definition, and treatment.

Attention should be paid to the processes of disinfection, sterilisation and cleaning of instruments, equipment, and environments. Longer intervals between appointments are necessary to provide enough time for environmental decontamination. To date, for the Brazilian MS there is no consensus on the use of substances that are common for mouth rinsing, such as hydrogen peroxide and chlorhexidine, 
impacting the reduction of viral load and / or decreasing professional contamination.

Dental care must take place in a decontaminated and ventilated environment with open windows, avoiding the use of air conditioning. It is important to remove items not related to dental care since they may accumulate microorganisms on their surfaces. During treatment, it is recommended that only patient and professionals who actually provide care remain at service location. Exceptions are guardians of underage patients, or companions of patients with disabling conditions.

Manual treatment techniques are priority, aiming at aerosol reduction, and aerosol producing equipment must be used together with high-power suction. The use of rubber dam is recommended to minimise the spread of fluids. When urgent care requires the use aerosols producing equipment, patients should be treated in the last service hours, whenever possible.

Following guidelines published by the Brazilian Federal Dental Council ${ }^{20}$ dental clinics must meet standard precaution requirements for all patients, regardless of whether COVID-19 infection is suspected or not:

a. surgical masks must be easily accessible to patients and instructions for their use must be provided;

b. alcohol $70 \%$ dispensers must be easily accessible to patients, from reception to the dental chair;

c. disposable tissues should be available for nasal hygiene, should patients need them;

d. professionals should thoroughly wash their hands with soap and water or rub them with $70 \%$ alcohol solution before and after contact with any patient, after removing gloves and after contact with blood and secretions. Face should be washed with soap and water when arriving at the clinic and between patients;

e. gloves must be used when there is risk of contact with blood and secretions or mucous membranes. They should be put on immediately before patient contact and removed immediately after treatment;

f. Goggles, approved masks, and impermeable gowns must be used when there is risk of contact with blood or secretions. They should protect eyes, mouth, nose, clothing and body surfaces

g. visual alerts can be placed in strategic locations (waiting areas, parking and elevators) to instruct patients on how to proceed when coughing or sneezing;

h. whenever possible, the dental clinic should have a comfortable private room where suspected COVID-19 patients could wait for their appointment.

\section{General considerations}

Immunity and viral evolution are interrelated key variables for the future of the pandemic and the consequent continuous development of oral health care protocols. Although recently developed COVID-19 vaccines are expected to produce immunity gradually and successively at local, national, and international levels, the disease is likely to remain a challenge for dental care. Given evidence that re-infection is unlikely in the short term ${ }^{21}$ there are two possible future scenarios: in the optimistic one immune response to SARS-CoV-2 is long lasting and, in populations with high disease incidence, new cases will be rare and self-contained; in the pessimistic scenario immunity lasts for only a few months, as seen for other human coronaviruses ${ }^{22}$ and we will have periodic disease outbreaks while most of the population remains susceptible.

Regardless of either scenario, it must be acknowledged that guidelines and policies directed to dental professionals and to oral health practice will have to be adapted to new data and accumulated knowledge on COVID-19, incorporating stakeholders in the permanent updating process. These interested parties could be grouped into: National political representatives (legislators, governors), public health agencies (ministry of health, social security agency, ministry of finance), labor (unions, dental associations), commercial/private for-profit, nonprofit (nongovernmental organizations, foundations), civil society, international/donors and oral health services users. ${ }^{23}$

Shortly after the publication of WHO's 2019 policy paper Building Bridges in Preparedness ${ }^{9}$ for pandemic influenza, COVID-19 has established a turning point 
in oral health care. Through several months of the pandemic, countries have continuously applied, reviewed, and updated their clinical recommendations and infection control strategies. Past and present epidemic and pandemic developments have increased the preparedness of dental schools, regulatory boards, scientific associations, government authorities, and oral health care providers to face past, present, and future biosafety challenges in the dental environment. All protocols and guidelines should be viewed as a dynamic document that will be updated frequently to adapt to ever-changing circumstances. Collect feedback from coworkers and key community partners is essential to identify any gaps in the contingency plan or needs for additional resources, supplies and equipment. DCHP should keep up to date to the current guidance of clinical practice during the pandemic to continue be able to deliver care to their patients and continuously revisit the risk-assessment to evaluate the effectiveness of emergency operations and communication plans.

\section{Acknowledgements}

Special thanks to the National Council for Scientific and Technological Development (CNPq), to the Higher Education Personnel Improvement Coordination (Capes) and to the Minas Gerais State Research Support Foundation (Fapemig).

\section{References}

1. Mattos FF, Pordeus IA. COVID-19: a new turning point for dental practice. Braz Oral Res. 2020;34:e085. https://doi.org/10.1590/1807-3107bor-2020.vol34.0085

2. Pereira LJ, Pereira CV, Murata RM, Pardi V, Pereira-Dourado SM. Biological and social aspects of Coronavirus Disease 2019 (COVID-19) related to oral health. Brazilian Oral Research. 2020;34:e041. https://doi.org/10.1590/1807-3107bor-2020.vol34.0041

3. Meng L, Hua F, Bian Z. Coronavirus Disease 2019 (COVID-19): emerging and future challenges for dental and oral medicine. J Dent Res. 2020 May;99(5):481-7. https://doi.org/10.1177/0022034520914246

4. Centers of Disease Control and Prevention - CDC. Recommendation: Postpone non-urgent dental procedures, surgeries, and visits. centers of disease control and prevention. 2020 [cited 2020 Mar 31]. Available from: https://www.cdc.gov/oralhealth/infectioncontrol/ statement-COVID.html

5. American Dental Association - ADA. ADA recommending dentists postpone elective procedures. Chicago: American Dental Assocaition; 2020 Mar 16 [cited 2020 Mar 31]. Available from: https://www.ada.org/en/publications/ada-news/2020-archive/march/adarecommending-dentists-postpone-elective-procedures

6. American Dental Association - ADA. Return to work interim guidance toolkit overview. Chicago: American Dental Assocaition; 2020 [cited 2020 Oct 5]. Available from: https://pages.ada.org/return-to-work-toolkit-american-dental-association

7. Centers of Disease Control and Prevention - CDC. Guidance for dental settings. 2020 [cited 2020 Oct 1]. Available from: https://www.cdc.gov/coronavirus/2019-ncov/hcp/dental-settings.html

8. Hopman J, Allegranzi B, Mehtar S. Managing COVID-19 in Low- and middle-income countries. JAMA. 2020 Apr;323(16):1549-50. https://doi.org/10.1001/jama.2020.4169

9. World Health Organization - WHO. Building bridges in preparedness. In: Report of a stakeholder consultation on national health security and pandemic influenza preparedness planning, , Accra, Ghana, 2017 Dec. 5-7. Geneva: World Health Organization; 2019.

10. Peng Z, Song W, Ding Z, Guan $Q$, Yang $X, X \cup Q$, et al. Linking key intervention timings to rapid declining effective reproduction number to quantify lessons against COVID-19. Front Med. 2020 Oct;14(5):623-9. https://doi.org/10.1007/s11684-020-0788-3

11. Thanh Le T, Andreadakis Z, Kumar A, Gómez Román R, Tollefsen S, Saville M, et al. The COVID-19 vaccine development landscape. Nat Rev Drug Discov. 2020 May;19(5):305-6. https://doi.org/10.1038/d41573-020-00073-5

12. Centers of Disease Control and Prevention-CDC. Non-COVID-19 care. 2020 June 30 [cited 2020 Oct 1]. Available from: https://www.cdc.gov/coronavirus/2019-ncov/hcp/framework-non-COVID-care.html

13. Centers of Disease Control and Prevention - CDC. Interim infection prevention and control recommendations for healthcare personnel during the Coronavirus disease 2019 (COVID-19) pandemic. 2020 [cited 2020 Oct 1]. Available from: https://www.cdc.gov/ coronavirus/2019-ncov/hcp/infection-control-recommendations.html

14. Kohn WG, Collins AS, Cleveland JL, Harte JA, Eklund KJ, Malvitz DM. Guidelines for infection control in dental health-care settings 2003. MMWR. 2020 Dec [cited 2020 Oct 2];52(RR-17). Available from: https://www.cdc.gov/mmwr/PDF/rr/rr5217.pdf 
15. Estrich CG, Mikkelsen M, Morrissey R, Geisinger ML, loannidou E, Vujicic M, et al. Estimating COVID-19 prevalence and infection control practices among US dentists. J Am Dent Assoc. 2020 Nov;151(11):815-24. https://doi.org/10.1016/i.adaj.2020.09.005

16. American Dental Association - ADA. ADA interim guidance for minimizing risk of COVID-19 transmission. Chicago, 2020 [cited 2020 Oct 5]. Available from: https://www.ada.org/en/publications/ada-news/2020-archive/april/ada-releases-interim-guidance-onminimizing-covid-19-transmission-risk-when-treating-emergencies

17. American Dental Association - ADA. What to do if someone on your staff tests positive for COVID-19. Chicago: American Dental Assocaition; 2020 [cited 2020 Oct 8]. Available from: https://success.ada.org/ /media/CPS/Files/COVID/A_Positive_COVID-19_Test_ Result_On_Your_Staff.pdf

18. Agência Nacional de Vigilância Sanitária - Anvisa. Nota Técnica No 04/2020 GVIMS/GGTES/ANVISA No 04/2020, de 31 de março de 2020. Orientações para serviços de saúde: medidas de prevenção e controle que devem ser adotadas durante a assistência aos casos suspeitos ou confirmados de infecção pelo novo Coronavírus (SARS-CoV-2). Rio de Janeiro: Agência Nacional de Vigilância Sanitária; 2020 [cited 2020 Oct 16]. Available from: https://www20.anvisa.gov.br/segurancadopaciente/index.php/alertas/item/notatecnica-n-04-2020-gvims-ggtes-anvisa-atualizada

19. Ministério da Saúde (BR). Nota técnica No 16/2020-CGSB/DESF/SAPS/MS. COVID-19 e atendimento odontológico no SUS. Brasília, DF: Ministério da Saúde; 2020 [cited 2020 Oct 16]. Available from: http://www.crosp.org.br/uploads/arquivo/295c9c14409db20cb63c $862 \mathrm{bb07ce0e4.pdf}$

20. Tomé G, Bernardes SR, Guandalini S, Guimarães MC. Manual de boas práticas em biossegurança para ambientes odontológicos. Brasília, DF: Conselho Federal de Odontologia; 2020 [cited 2020 Oct 16]. Available from: https://website.cfo.org.br/wp-content/ uploads/2020/04/cfo-lança-Manual-de-Boas-Práticas-em-Biossegurança-para-Ambientes-Odontologicos.pdf

21. Kellam P, Barclay W. The dynamics of humoral immune responses following SARS-CoV-2 infection and the potential for reinfection. J Gen Virol. 2020 Aug;101(8):791-7. https://doi.org/10.1099/igv.0.001439

22. Edridge AW, Kaczorowska J, Hoste AC, Bakker M, Klein M, Loens K, et al. Seasonal coronavirus protective immunity is short-lasting. Nat Med. 2020 Nov;26(11):1691-3. https://doi.org/10.1038/s41591-020-1083-1

23. Brugha R, Varvasovszky Z. Stakeholder analysis: a review. Health Policy Plan. 2000 Sep;15(3):239-46. https://doi.org/10.1093/heapol/15.3.239 